\title{
Path Diversity Gain with Network Coding and Multipath Transmission in Wireless Mesh Networks
}

\author{
Nikolaos Pappas, Vasilios A. Siris, Apostolos Traganitis \\ Institute of Computer Science, Foundation for Research and Technology - Hellas (FORTH) \\ P.O. Box 1385, GR 71110 Heraklion, Crete, Greece \\ \{npapas,vsiris,tragani\}@ics.forth.gr
}

\begin{abstract}
In this paper we investigate the performance that can be achieved by exploiting path diversity through multipath forwarding together with redundancy through linear network coding, in wireless mesh networks with directional links. A key contribution of the paper is to capture the tradeoff between packet delay and throughput achieved by combining multipath forwarding and network coding, and compare this tradeoff with that of simple multipath routing where different flows follow different paths, the transmission of multiple copies of packets over multiple paths, and single path routing. The analytical framework considers the case of end-to-end retransmission for achieving reliability, and is generalized for an arbitrary number of paths and hops. We also derive the minimum and maximum number of coded packets that are needed at the receiver to retrieve all packets sent by the sender; this can be used to obtain a lower and upper bound for the delay in the case of linear network coding with multipath forwarding.
\end{abstract}

\section{INTRODUCTION}

The core notion of network coding introduced in [1] is to allow and encourage mixing of data at intermediate network nodes. Network coding is a generalization of the traditional store and forward technique. Most of the theoretical results in network coding are for multicast but the vast majority of Internet traffic is unicast. An application of network coding to wireless environments has to address multiple unicast flows, if it has any chance of being used. In particular, with multicast, all receivers want all packets. Thus intermediate nodes can encode any packets together, without worrying about decoding which will happen eventually at the destinations.

We consider unicast flows in a multi-hop wireless mesh network with lossy directional links. In such networks the largest percentage of uplink traffic is destined for or originates from a gateway interconnecting the mesh network to a wired network. Moreover, a mesh node can provide access to multiple clients. Hence, the uplink traffic from these clients that is destined to the same gateway can be coded at the mesh node, and decoded at the gateway. Similarly, downlink traffic destined for the clients of the same mesh node can be coded at the gateway and decoded at the mesh node.

This work was supported in part by the European Commission in the 7th Framework Programme through project EU-MESH (Enhanced, Ubiquitous, and Dependable Broadband Access using MESH Networks), ICT-215320, http://www.eu-mesh.eu

N. Pappas and A. Traganitis are also with the University of Crete. V. A. Siris is also with the Athens University of Economics and Business.
The goal of this paper is to investigate the performance that can be achieved by exploiting path diversity through multipath forwarding and redundancy through network coding. Specifically, we compare the performance and tradeoff in terms of packet delay and throughput achieved by combining multipath forwarding and network coding, with that of simple multipath routing of different flows (which achieves the highest throughput), the transmission of multiple copies of a single flow over multiple paths (which achieves the highest redundancy and the least delay), and traditional single path routing.

The idea of using redundancy is central in channel coding theory. In this work we use redundant paths to send coded packets in order to recover the loss of information using packets from another path, thus decreasing the delay. The work in [2] uses path diversity for fast recovery from link outages. The work in [3] introduces error correcting network coding as a generalization of classical error correcting codes. The work in [4] studies the coding delay in packet networks that support network coding. The authors in [5] propose efficient algorithms for the construction of robust network codes for multicast connections. The goal of this work is to to provide instantaneous recovery from single edge failures. The work in [6] presents an approach for designing network codes by considering path failures in the network instead of edge failures. There is a lot of work for opportunistic routing in wireless mesh networks, with or without network coding. COPE [7], MORE [8] and $\mathrm{MC}^{2}$ [9] investigate network coding with opportunistic routing in wireless networks with broadcast transmissions, focusing exclusively on the throughput improvements. ExOR [10] and ROMER [11] investigate opportunistic routing in broadcast wireless networks without network coding. Moreover, these works also focus on the throughput improvements, except [11] which also considers the packet delivery ratio. The work of [12] considers diversity coding, and investigates the allocation of data to multiple paths that maximizes the probability of successful reception. The work of [13] extends the previous work, in the case where the failure probabilities are different for different paths, and when the paths are not necessarily independent.

Our contribution and a key difference with the previous works is that we study the delay and throughput tradeoff and compare network coding with other transmission schemes such as single path, multipath and multicopy. We study the average 


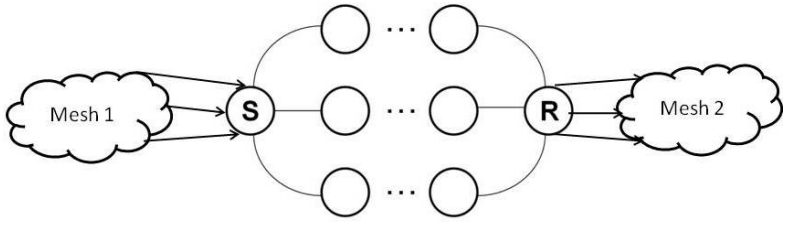

Fig. 1. Simple three path network

delay per packet and the throughput achieved, disregarding the queueing delay at the sender, the encoding and decoding delays, and the ACK transmission delays.

The model we assume is a one-source unicast acyclic network with lossy links. The nodes inside the network (except the source and the destination) act as relays, do not decode the information but simply forward coded packets that have been previously received from the source or the previous node. This allows for uncoordinated, low-complexity processing at the nodes.

The analytical framework presented in this paper considers the case of end-to-end retransmission for achieving reliability, and is generalized for an arbitrary number of paths and hops. We also derive the minimum and maximum number of coded packets that are needed at the receiver to retrieve all packets sent by the sender; this can be used to obtain a lower and upper bound for the delay in the case of linear network coding with multipath forwarding. The application of linear network coding results in the considerable reduction of the computational complexity at the nodes. An interesting conclusion that comes out from this work is that network coding gives us an advantage in terms of delay-throughput trade-off. We will see that when the number of paths increasing network coding unfolds it advantages comparing to other routing schemes.

The paper is organized as follows: Section II presents the analytical model for the throughput and delay in the case of end-to-end retransmissions, for three and for $2^{k}-1$ path networks. Section III presents numerical results based on the previous models, and section IV concludes the paper.

\section{AnAlytical Model}

In this section we are presenting the analytical model for the throughput and delay in the case of end-to-end retransmissions. We initially consider the simple model shown in figure 1, which contains three paths from node $\mathrm{S}$ to node $\mathrm{R}$, then we extend to $2^{k}-1$ paths.

Recall from the previous section that node $\mathrm{S}$ can be a wired network gateway and node $\mathrm{R}$ can be a mesh node connecting multiple wireless clients or vice versa. Each path has $n$ hops.

We consider the following approaches for transmitting packets from $\mathrm{S}$ to $\mathrm{R}$ : With single path routing, packets from all flows follow the same single path, leaving the other paths unutilized. With simple multipath routing, all the paths are used to transmit packets from different flows; the packets belonging to the same flow follow the same path. Another alternative is to transmit copies of packets belonging to a single flow on all the links; we call this scheme multicopy.

Finally the last scheme combines multipath with network coding. For the three path network shown in figure 1 node $\mathrm{S}$ sends three linear combinations $x_{i} a+y_{i} b$ for $i=1,2,3$, of packets $a$ and $b$ along the three paths; the receiver needs to receive at least two linear independent of these combinations in order to decode the packets, and retrieve the original two packets $a$ and $b$.

We assume that the probability of a packet error on each link is the same, and equal to $e$. If a path consists of $n$ hops, then the probability of a packet correctly reaching node $\mathrm{R}$ is $P_{c}=(1-e)^{n}$; then $P_{e}=1-P_{c}$ is the probability of a packet error along the whole path.

Next we compute the packet delay and throughput achieved by each of the forwarding schemes mentioned above. The packet delay $D$ is the delay for transmitting a packet from the $\mathrm{S}$ to $\mathrm{R}$, when the packet is at the head of the transmission queue at $\mathrm{S}$, i.e., we do not include in $D$ the queuing delay at $\mathrm{S}$, and we assume there is no congestion, hence no queuing delay, in the intermediate nodes. We also assume that the transmission delay of each hop is one. Moreover, if a packet is not correctly received by $\mathrm{R}$, it is retransmitted by node $\mathrm{S}$; we disregard the delay for transmitting ACKs back from R to $\mathrm{S}$.

\section{A. Analysis for a three-path network with $n$ hops}

1) Single Path: The average delay is given by

$$
D_{s p}=\left(1-P_{e}\right) n+P_{e}\left(n+D_{s p}\right) \Leftrightarrow D_{s p}=\frac{n}{1-P_{e}},
$$

and the throughput is

$$
T h r_{s p}=\frac{1}{D_{s p}}=\frac{1-P_{e}}{n} .
$$

2) Multipath: Multipath has the same delay as single path

$$
D_{m p}=D_{s p},
$$

and its throughput is three times the throughput of single path

$$
T h r_{m p}=3 T h r_{s p} .
$$

3) Multicopy: The delay and throughput are

$$
D_{\text {mcop }}=\frac{n}{1-P_{e}^{3}}, \quad T h r_{\text {mcop }}=\frac{1}{D_{\text {mcop }}} .
$$

4) Multipath with Network Coding: The delay $D_{n c}$ is the average delay to receive at least two of the three independent linear combinations sent by node $\mathrm{S}$ :

$$
\begin{array}{r}
D_{n c}=\left(1-P_{e}\right)^{3} n+3 P_{e}\left(1-P_{e}\right)^{2} n+ \\
+3 P_{e}^{2}\left(1-P_{e}\right)\left(n+D_{1}\right)+P_{e}^{3}\left(n+D_{n c}\right)
\end{array}
$$

where

$$
D_{1}=\left(1-P_{e}^{2}\right) n+P_{e}^{2}\left(n+D_{1}\right) .
$$

The first term in the expression for $D_{n c}$ corresponds to the case of correct transmission on all three paths. The second term 
corresponds to the case of an error in one of the three paths. The third term corresponds to the case of errors in two of the three paths, hence there is an additional delay $D_{1}$ to receive one more linear combination. The last term corresponds to the case where there were errors on all three paths. Since in the time interval $D_{n c}$ node $\mathrm{R}$ receives two data packets, the average throughput is given by

$$
T h r_{n c}=\frac{2}{D_{n c}} .
$$

\section{B. Generalization for $2^{k}-1$ paths}

Here we extend the previous model to the case of $2^{k}-1$ paths,

1) Single Path:

$$
D_{s p}=\frac{n}{1-P_{e}}, \quad T h r_{s p}=\frac{1}{D_{s p}} .
$$

2) Multipath:

$$
D_{m p}=D_{s p}, \quad T h r_{m p}=\left(2^{k}-1\right) T h r_{s p} .
$$

3) Multicopy:

$$
D_{\text {mcop }}=\frac{n}{1-P_{e}^{2^{k}-1}}, \quad T h r_{\text {mcop }}=\frac{1}{D_{\text {mcop }}} .
$$

4) Multipath with Network Coding: We have $k$ packets to transmit through $2^{k}-1$ paths with $n$ hops each. We calculate the delay for the reception of at least $k$ from a set of $2^{k}-1$ packets. The delay to receive $k$ packets when we have already received $j$ packets is denoted by $D_{k, j}$. The delay we are interested in is $D_{n c}=D_{k, 0}$.

$$
\begin{gathered}
D_{k, j}=\sum_{i=k-j}^{2^{k}-j-1}\left(\begin{array}{c}
2^{k}-j-1 \\
i
\end{array}\right) P_{c}^{i} P_{e}^{2^{k}-i-j-1} n+ \\
+\sum_{i=0}^{k-j-1}\left(\begin{array}{c}
2^{k}-j-1 \\
i
\end{array}\right) P_{c}^{i} P_{e}^{2^{k}-i-j-1}\left(n+D_{k, i}\right), \\
T h r_{n c}=\frac{k}{D_{n c}} .
\end{gathered}
$$

\section{NUMERICAL EXPERIMENTS}

In this section we present arithmetic results based on the models in section II. Figures 2(a) and 2(b) show the delay throughput tradeoff for error probabilities 0.2 and 0.4 respectively for networks with three paths of one hop each. Multipath with network coding achieves delay which is smaller than single and multipath, but worst than multi-copy forwarding. The throughput achieved by multipath with network coding is better than that achieved by multicopy forwarding.

Error probabilities are assumed to be between 0.1 and 0.8 in the figures shown in current section. We want to compare the presented routing schemes in environments with heavy noise. Heavy noise in the wireless medium can be explained by interference, path losses and fading as it is well known.

Figure 3(a) shows that, as expected, the improvement in terms of lower delay which is achieved by multipath with

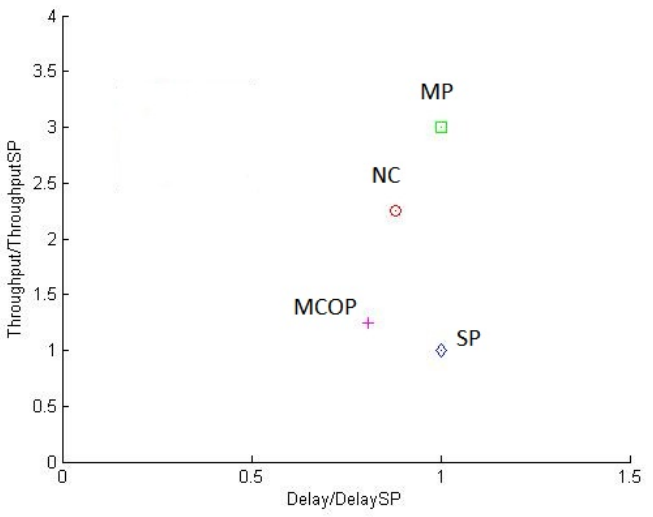

(a) $e=0.2$

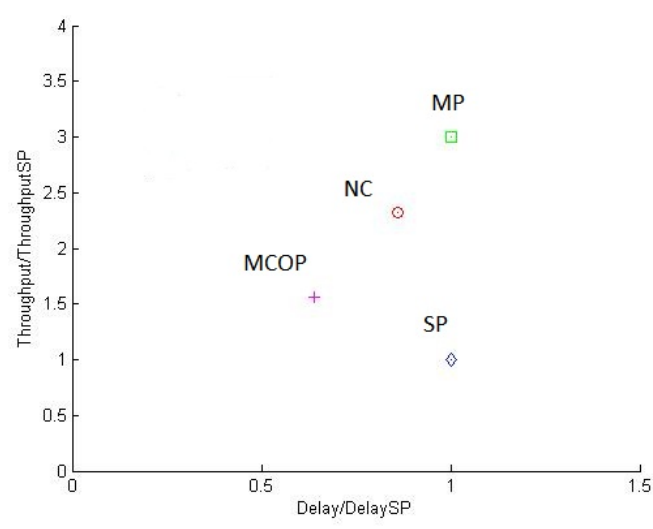

(b) $e=0.4$

Fig. 2. Delay-throughput tradeoff in the case of three paths with one hop each

network coding and multi-copy increases with increasing error probability. Regarding throughput, observe that a higher loss probability does not significantly affect the gains of multipath with network coding over single path forwarding, as much as it does for multicopy forwarding; this is also shown in figure 3(b).

Figures 4(a) and 4(b) show how the number of hops affects the throughput and delay. In particular, figure 4(a) shows that the improvement in terms of lower delay compared to single path forwarding increases with the number of hops, for both multipath forwarding with network coding and multicopy forwarding. Moreover, figure 4(b) shows that whereas for multipath forwarding with network coding, the throughput improvement compared to simple multipath forwarding remains relatively constant as the number of hops increase, for multicopy forwarding the throughput gain increases and after some number of hops, the gain with multicopy forwarding is higher compared to the gain with multipath forwarding with network coding. The above results for three paths indicate that the network coding delay gain over the single path and multipath schemes is about $15-20 \%$. On the other hand, multicopy forwarding is superior when the loss becomes large and for a large number of hops because of its higher redundancy. 


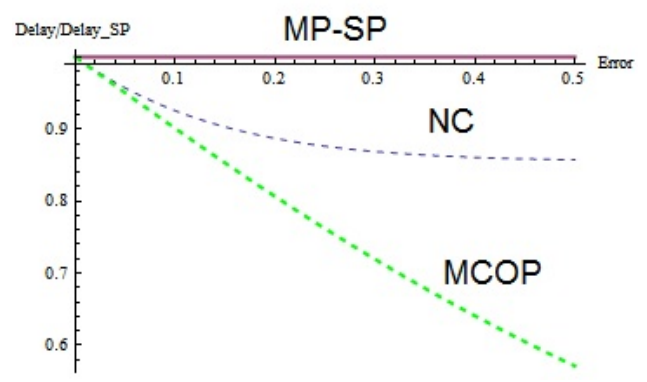

(a) $D / D_{s p}$ vs error probability

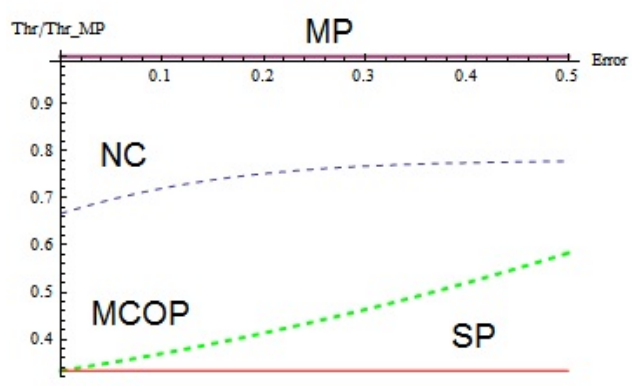

(b) $T h r / T h r_{m p}$ vs error probability

Fig. 3. Delay and throughput for different errors probabilities, in the case of three paths with one hop each

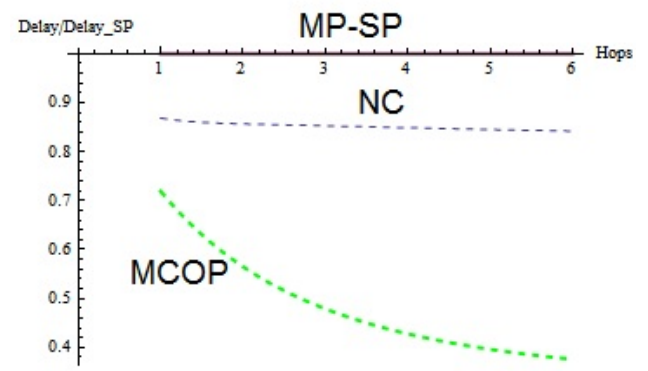

(a) $D / D_{s p}$ vs number of hops

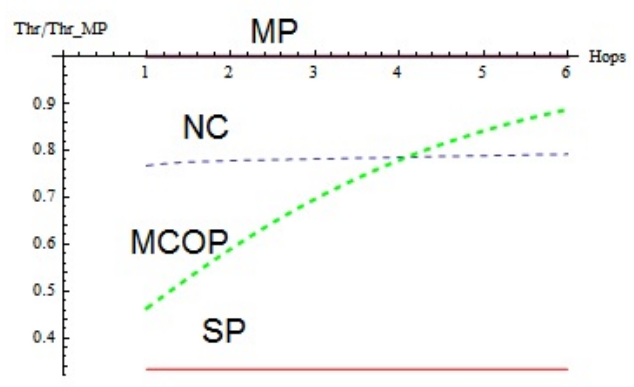

(b) $T h r / T h r_{m p}$ vs number of hops

Fig. 4. Delay and throughput for different number of hops, in the case of three paths and $e=0.3$

Figures 5(a) and 5(b) show the delay - throughput tradeoff for error probabilities 0.2 and 0.4 respectively for a network with seven paths, each with one hop. These figures include two graphs for network coding, one corresponding to the case of decoding after receiving three linear combinations (which is denoted by NC-L) and one for decoding after receiving

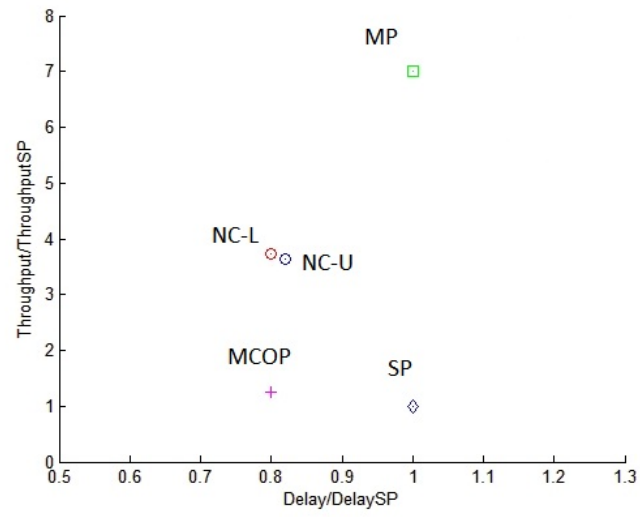

(a) $e=0.2$

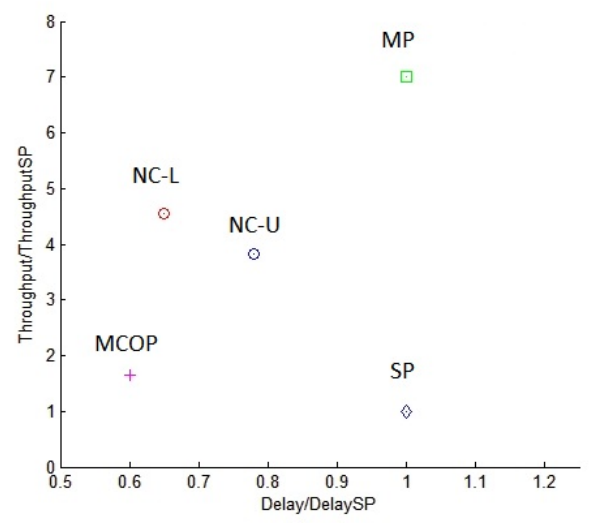

(b) $e=0.4$

Fig. 5. Delay-thoughput tradeoff in the case of seven paths

four (which is denoted by NC-U); These numbers represent the lower and upper bound of the number of coded packets required to retrieve all packets at the receiver, as indicated by lemma A. Multipath with network coding achieves delay, which is better than single and multipath, but approaches the delay of multicopy forwarding. Comparison of Figures 5(a) and 5(b), with Figures 2(a) and 2(b) shows that the improvements of network coding increase as the number of paths increases. Also, the throughput achieved by multipath with network coding is better than that achieved by multicopy forwarding.

Figure 6(a) shows that, as in 3(a), the improvement in terms of lower delay which is achieved by multipath with network coding and multicopy increases with increasing error probability for the case of seven paths.

Figures 7(a) and 7(b) show how the number of hops affects the throughput and delay for a network with seven paths. In particular, figure 7(a) shows that the improvement in terms of lower delay compared to single path forwarding increases with the number of hops, for both multipath forwarding with network coding and multicopy forwarding. Moreover, figure 7(b) shows that whereas for multipath forwarding with network coding, the throughput improvement compared to simple multipath forwarding remains relatively constant as the number of hops increases, for multicopy forwarding the 


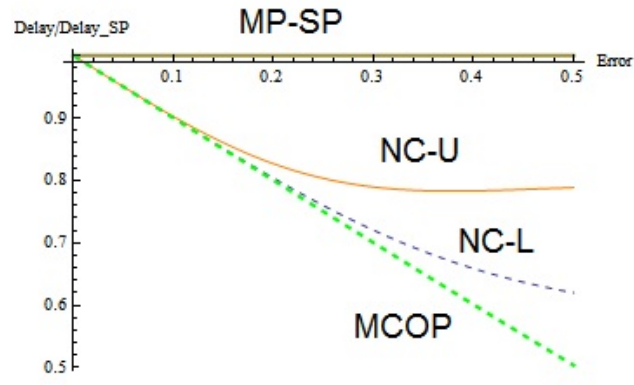

(a) $D / D_{s p}$ vs error probability

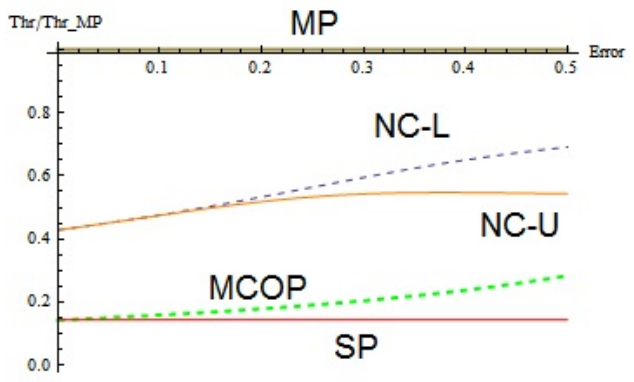

(b) $T h r / T h r_{m p}$ vs error probability

Fig. 6. Delay and throughput in the case of seven paths with one hop each

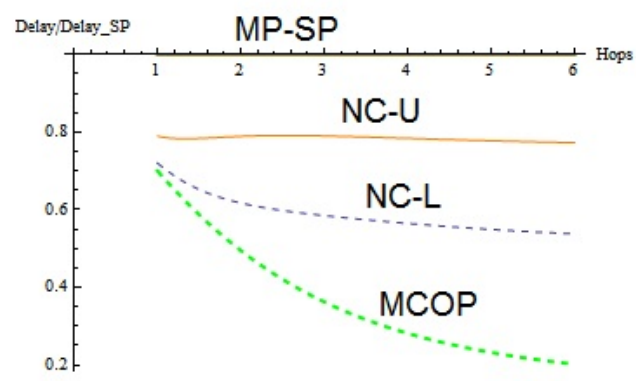

(a) $D / D_{s p}$ vs number of hops

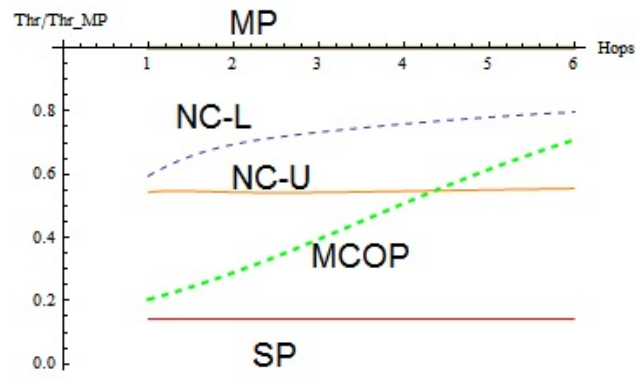

(b) $T h r / T h r_{m p}$ vs number of hops

Fig. 7. Delay and throughput for a different number of hops, in the case of seven paths and $e=0.3$

throughput gain increases and after some number of hops, the gain with multicopy forwarding is higher compared to the gain with multipath forwarding with network coding. The above results for seven paths suggest that the network coding delay gain compare to single path and multipath forwarding is about $20-40 \%$. On the other hand, in the case of seven paths, the delay with network coding is close to the delay with multicopy forwarding.

\section{CONCLUSion}

In this paper we investigated the performance and reliability that can be achieved by exploiting path diversity through multipath forwarding together with redundancy through network coding, when end-to-end retransmissions are used for achieving reliable packet transmission. The work in this paper is at very fundamental level and it is not supposed to provide blueprints for a real network, however it helps understanding about network coding and its impact on redundancy and the trade-off among other routing schemes. We compared the performance and tradeoff in terms of packet delay and throughput achieved by combining multipath forwarding and network coding, with that of simple multipath routing of different flows, transmission of multiple copies of a single flow over multiple paths, and single path routing. We saw that network coding decreases the delay that is needed for the transmission of a packet compared with multipath and traditional single path forwarding, achieving a delay-throughput balance that lies between the corresponding performance of simple multipath and multicopy forwarding, which sends the same packet across all available paths. Another important result is that as the number of available paths increases, the gain from network coding also increases.

Future work will investigate the delay - throughput tradeoffs in the case of hop-by-hop retransmissions. Another important issue is the correlation of losses among the paths, it is interesting also the study of paths that contain links with bursty errors. Initial results indicate that in the case of networks that have paths with common links, the advantages of network coding are more pronounced. The analysis done in the present paper will serve as a guideline for a more general network model including the previous considerations.

\section{REFERENCES}

[1] R. Ahlswede, N. Cai, S. R. Li, and R. W. Yeung, "Network information flow," in IEEE Transactions on Information Theory, 2000, pp. 12041216.

[2] Ayanoglu, Chih-Lin I, R.D. Gitlin, and J.E. Mazo, "Diversity coding for transparent self-healing and fault-tolerantcommunication networks," in IEEE Transactions on Communications, 1993, pp. 1677 - 1686.

[3] R. W. Yeung and N. Cai, "Network error correction, part i: Basic concepts and upper bounds," in Communications in Information and Systems, 2006, pp. $19-36$.

[4] D. Lun, M. Medard, R. Koetter, and M. Effros, "Further results on coding for reliable communication over packet networks," in ISIT, 2005.

[5] Graham Booker and Alex Sprintson, "Design of efficient robust network codes for multicast connections," in ISIT, 2009.

[6] H. Bahramgiri and F. Lahouti, "Robust network coding against path failures," in IET Communications, 2010, pp. 272-284.

[7] Sachin Katti, Dina Katabi, Wenjun Hu, Hariharan Rahul, and Muriel Medard, "The importance of being opportunistic: Practical network coding for wireless environments," in Allerton, 2005.

[8] Szymon Chachulski, Michael Jennings, Sachin Katti, and Dina Katabi, "Trading structure for randomness in wireless opportunistic routing," in ACM SIGCOMM, 2007.

[9] Christos Gkantsidis, Wenjun Hu, Peter Key, Bozidar Radunovic, Steluta Gheorghiu, and Pablo Rodriguez, "Multipath code casting for wireless mesh networks," in CoNext, 2007.

[10] S. Biswas and R. Morris, "Opportunistic routing in multi-hop wireless networks," in ACM SIGCOMM, 2005.

[11] Yuan Yuan, Starsky H. Y. Wong, Songwu Lu, and William Arbuagh, "Romer: Resilient opportunistic mesh routing for wireless mesh networks," in Proceedings of IEEE WiMesh, 2005. 
[12] A. Tsirigos and Z.J. Haas, "Analysis of multipath routing, part 1: The effect of packet delivery ratio," in IEEE Transactions on Wireless Communications vol.3 no. 1, 2004, pp. 138-146.

[13] A. Tsirigos and Z.J. Haas, "Analysis of multipath routing, part 2: Mitigation of the effects of frequently changing topologies," in IEEE Transactions on Wireless Communications vol.3 no. 2, 2004, pp. 500511.

\section{APPENDIX A \\ LEMMA}

In this appendix we give a Lemma that identifies the minimum and the maximum number of linear combinations needed in order to retrieve all the packets when we use network coding.

Lemma 1: Consider a linear $n$ dimensional vector space $G F(2)^{n}$. We need exactly $n$ linear independent vectors and at most $\left\lceil\left(2^{n}-1\right) / 2\right\rceil=\left\lceil 2^{n-1}-1 / 2\right\rceil=2^{n-1}$ different vectors(not independent) (excluding the zero vector) in order to reconstruct the vector space.

Proof: It is obvious that we need exactly $n$ linear independent vectors, but with a random selection of $n$ linear combinations there is a possibility that the vectors cannot span the space due to linear dependency. The vectors in $G F(2)^{n}$ have $n$ coordinates and there are $2^{n}-1$ vectors (excluding the zero one). If we choose $2^{n-1}-1$ we can span an $n-1$ dimensional subspace of $G F(2)^{n}$ space; this means that we have a collection of vectors that in total have $n-1$ coordinates with value 1 . So, we need one more coordinate to be different than zero in the previous collection of $2^{n-1}-1$, we choose another one from the pool of the $2^{n}-1$ vectors. Now it is obvious from Pigeonhole principle that we are able to span the $n$ dimensional space.

For example, if $n=3$, we need three linear independent vectors in order to construct the vector space, and any collection of $4=2^{3-1}$ different vectors spans the three dimensional vector space.

\section{APPENDIX B \\ GENERALIZATION FOR $2^{k}-1$ PATHS WITH DIFFERENT ERROR PROBABILITIES}

In this appendix we extend the model in section II-B to the case of paths with different error characteristics. With this extension we are able to encapsulate to our study notions as congestion that exist in real networks. We denote $P_{e, i}$ the probability of a transmission error at path $i$.

1) Single path:

$$
\begin{gathered}
D_{s p}=\frac{n}{1-\min _{i} P_{e, i}} \\
T h r_{s p}=\frac{1}{D_{s p}}
\end{gathered}
$$

\section{2) Multipath:}

$$
D_{m p}=\frac{1}{2^{k}-1} \sum_{i=1}^{2^{k}-1} \frac{n}{1-P_{e, i}}
$$

$$
T h r_{m p}=2^{k}-1-\sum_{i=1}^{2^{k}-1} P_{e, i}
$$

3) Multicopy:

$$
\begin{gathered}
D_{\text {mcop }}=n /\left(1-\prod_{i=1}^{2^{k}-1} P_{e_{i}}\right) \\
T h r_{m c}=\frac{1}{D_{m c}}
\end{gathered}
$$

4) Multipath with Network Coding: If $N=2^{k}-1$ is the number of paths and $K$ is the number of linear combinations necessary to decode correctly the original $k$ packets, then from lemma A we must have $k \leq K \leq 2^{k-1}$. The delay to receive these $K$ linear combinations is

$$
\begin{aligned}
& D_{n c}=\prod_{i=1}^{N}\left(1-P_{e, i}\right) n+\sum_{i=1}^{N} P_{e, i} \prod_{\substack{j=1 \\
j \neq i}}^{N}\left(1-P_{e, i}\right) n+ \\
& +\sum_{i=1}^{N} \sum_{\substack{j=1 \\
j \neq i}}^{N} P_{e, i} P_{e, j} \prod_{\substack{k=1 \\
k \neq i, j}}^{N}\left(1-P_{e, k}\right) n+\ldots \\
& +\sum_{i_{1}=1}^{N} \ldots \sum_{\substack{i_{N}-K=1 \\
i_{N-K} \neq i_{1}, \ldots, i_{N-K-1}}}^{N} \prod_{k=1}^{N-K} P_{e, i_{k}} \prod_{\substack{j=1 \\
j \neq i_{1}, \ldots, i_{N-K}}}^{N}\left(1-P_{e, j}\right) n+ \\
& +\sum_{i=1}^{N}\left(1-P_{e, i}\right) \prod_{\substack{j=1 \\
j \neq i}}^{N} P_{e, j}\left(n+D_{i}\right)+ \\
& +\sum_{i=1}^{N} \sum_{\substack{j=1 \\
j \neq i}}^{N}\left(1-P_{e, i}\right)\left(1-P_{e, j}\right) \prod_{\substack{k=1 \\
k \neq i, j}}^{N} P_{e, k}\left(n+D_{i, j}\right)+\ldots \\
& +\sum_{i_{1}=1}^{N} \ldots \sum_{\substack{i_{K-1}=1 \\
i_{K}, \ldots=1 \\
i_{1}, \ldots, i_{K-2}}}^{N} \prod_{\substack{1 \neq 1 \\
i_{1}, \ldots, i_{K-1}}}^{K-1} \prod_{\substack{j=1 \\
j \neq}}^{N} P_{e, j}\left(1-P_{e, i_{k}}\right)\left(n+D_{i_{1}, \ldots, i_{K-1}}\right)+ \\
& +\prod_{i=1}^{N} P_{e, i}\left(n+D_{n c}\right)
\end{aligned}
$$

where $D_{i_{1}, \ldots, i_{j}}$ is the delay to receive the additional $K-$ $j$ linear combinations after receiving $j$ linear combinations. The calculation of this delay is similar to the calculations in the preceding sections. Note that when a packet needs to be retransmitted, it follows the same path as the path of the initial transmission attempt. An interesting extension is to retransmit packets using the paths with the smallest error probability. Finally, the throughput is given by

$$
T h r_{n c}=\frac{k}{D_{n c}} .
$$

